\title{
The Fourth AMS Conference on Mesoscale Processes 25-29 June 1990, Boulder, Colorado
}

\author{
Daniel Keyser \\ Program Chairman, Department of Atmospheric Science, \\ State University of New York at Albany, Albany, NY 12222
}

\section{Introduction}

The Fourth AMS Conference on Mesoscale Processes was held at the Clarion Harvest House in Boulder, Colorado, 25-29 June 1990, in conjunction with the Fifth AMS Conference on Mountain Meteorology. The conference provided an international forum for portraying the contemporary landscape of the discipline of mesoscale meteorology, in particular the status of basic understanding of mesoscale phenomena and processes from observational, numericalmodeling, and theoretical points of view. Toward this end, contributions were organized among sessions dealing with: mesoscale aspects of extratropical cyclones; mesoscale cyclonic vortices; mesoscale predictability; fronts and frontogenesis; banded precipitation features in fronts and cyclones; gravity waves; mesoscale instabilities and turbulence; squall lines; mesoscale convective systems; momentum transport by convection; mesoscale phenomena influenced by topography; thermally and mechanically forced flows; and boundary-layer circulations and cloud distributions. Presentations on these topics are summarized below from reports submitted by the session chairs.

\section{Session summaries}

a. Session 1. Extratropical cyclones: Observations, modeling and theory (Frederick Sanders, chair)

M. Shapiro presented, from observations, a conceptual model of a developing frontal cyclone that departed from the traditional Norwegian model in some respects. The front was fractured just south of the center, leaving a warm front running from ahead, through the cyclone center, and then out the rear. Maximum temperature contrast was along this warm front. The informal appellation, "T-Bone," was sug-

C1991 American Meteorological Society gested for this configuration. He then showed the results of a 20-km mesh-length simulation of the storm documented during Intensive Observing Period \#4 (IOP-4) of the Experiment on Rapidly Intensifying Cyclones over the Atlantic (ERICA). This particular storm displayed much of the structure of the conceptual model. Close examination of observational material showed, however, that within this mesoscale frontal structure there was variability on even smaller scales, yet to be understood or modeled numerically.

$P$. Neiman and M. Shapiro presented a synoptic overview of the ERICA IOP-4 storm and discussed mesoscale details disclosed by low-level aircraft traverses of this storm. The "T-Bone" structure was shown in the equivalent potential temperature $\left(\theta_{\mathrm{e}}\right)$ field, as was an intense, small warm core. The chairperson suggested that the pattern was not significantly different from that of the "bent-back occlusion" in the Norwegian lore. There were questions concerning the relative roles of horizontal advection and heat flux from the sea surface in the development of the warm core. This important issue has not as yet been examined fully.

C. Kreitzberg presented evidence, from the chairperson's surface analyses, that the cyclogenesis in ERICA IOPs-1-5, as well as in IOPs-6-8, occurred in close association with the strong gradient of seasurface temperature along the NW wall of the Gulf Stream. The chairperson took issue with the interpretation of the complex development in IOP-2. The speaker identified the final spectacular intensification with a cyclone growing along the gradient and absorbing other cyclonic activity to the south, while the chairperson insisted that a cyclone somewhat to the south was the one that intensified, absorbing the Gulf Stream center. There is no doubt, however, about the importance of the Gulf Stream.

J. Gyakum showed detailed analyses of the Presidents' Day Storm of 1979, in which the forcing associated with a powerful oncoming upper-level distur- 
bance overspread a small pre-existing surface cyclone of independent source. The resulting extraordinary intensification could be viewed as the response of surface air with high initial vorticity to the convergence associated with the approaching upper trough. In the vorticity equation, the absolute vorticity appears as a coefficient multiplying the divergence term. In response to a question about reliability of details of the surface analysis, the speaker pointed to good data coverage and continuity. In response to an observation that the wind field around the initial surface cyclone was asymmetric, the chairperson commented that extreme asymmetry in gale-force, apparently supergeostrophic, southwesterly winds just off the Carolina coast characterized the initiation of the surface storm in ERICA IOP-4.

W. Nuss contrasted two consecutive surface cyclones propagating eastward along the strong seasurface temperature gradient of the Kuroshio. Upperlevel forcing was comparable in the two cases, but the earlier cyclone deepened only moderately while the cyclone three days later intensified explosively. It was argued that the key to the difference lay in the initial low-level temperature field. In the modest cyclone, the low-level southeasterly flow transported relatively warm air northeastward in the path of the cyclone, where heat flux from air to sea tended to stabilize the surface layers. In the strong storm, easterly surface winds ahead of the center maintained cold air over warm water as the cyclone approached, with heating by the sea in the region ahead of and near the center.

W. Lapenta and N. Seaman showed diagnoses of the results of a detailed short-term numerical prediction of a moderately intensifying coastal cyclone. Sensitivity of occurrence and position of the coastal low to elimination of 1) latent heating and of 2) the seasurface temperature structure associated with the Gulf Stream was considerable. Changing the intensity of the upper-level jet core had relatively little effect on development near the surface. Nevertheless, in response to a question why this cyclone failed to develop explosively despite the presence of many of the familiar ingredients, the speaker judged that the relatively low overall baroclinicity was responsible. M. Shapiro noted the absence of strong upper-level isentropic potential vorticity (IPV) advection.

A semigeostrophic formulation was used by Y.-L. Lin to study the development of a shallow surface disturbance in the presence of the Gulf Stream heat source and a mountain ridge to the west. To keep the growing disturbance over the heat source, it was necessary to postulate a reversal of wind in the vertical in the two-dimensional basic state. The semigeostrophic formulation was used to evaluate the effects of ageostrophic advection. The result, after $24 \mathrm{~h}$ of calculation, shows a cyclone near the Gulf Stream edge. This result is similar to an earlier one derived from a quasi-geostrophic model, but the low is somewhat deeper and eccentric, with maximum gradient in the SW quadrant where ageostrophic warm advection is largest.

M. Hedley and M. Yau presented calculations with an anelastic model characterized by $10-\mathrm{km}$ horizontal and $1-\mathrm{km}$ vertical resolution, explicit precipitation physics, and parameterized surface fluxes of heat and moisture. This fine-mesh area was nested within a much larger area of progressively decreasing horizontal resolution. When a very substantial perturbation of sea-surface temperature was used (said to be similar to that for ERICA IOP-2), a 50-mb deepening during the last $24 \mathrm{~h}$ of a 30 -h calculation was obtained, with realistic asymmetric structure in the fields of pressure, temperature, and cloud water. Removal of the seasurface temperature perturbation led to the simulation of an innocuous, weaker cyclone, devoid of prominent mesoscale structure. Asked to explain the real-atmosphere occurrence of spectacular cyclogenesis in cases of approximately uniform gradient of sea-surface temperature, the speaker suggested that inhomogeneities of static stability produced by the surface anomaly specified in his calculation may have been a result of prior history in the case of the real atmosphere.

C. Thorncroft described a study of the interaction of upper-level anomalies of potential vorticity (PV) with surface anomalies of potential temperature. The model is two-dimensional and semigeostrophic, an idealization designed to study earlier results of a three-dimensional primitive-equation model, in which surface development occurred not only expectedly downstream from an initial PV perturbation but also unexpectedly upstream, with accompanying PV perturbation developing subsequently. The idealized results showed rapid shearing away of the initial PV anomaly, refusal to lock on to the surface baroclinic zone, and downward transport of high PV values to the middle or lower troposphere. Upstream disturbances developed but were dominated by surface temperature anomalies. In response to a query, the chairperson commented that he had not seen disturbances of the upstream type. Interpretation of the results in terms of the real atmosphere, however, was restricted by the two-dimensionality of the model.

C. Bishop focused on the differences between the coupling of sinusoidal upper- and lower-level disturbances and that of localized upper- and lower-level disturbances in an Eady-type model. The main point was that mathematical analysis of uniform PV flows shows that although upper-level sinusoidal disturbances can be expected to lock on to lower-level disturbances, such an expectation is not necessarily 
valid for isolated upper-level disturbances lying on $\mathrm{PV}$ gradients. The reason is that much of the energy of localized disturbances is contained in the (baroclinically) neutral spectrum of wavelengths. The group velocity of these waves is approximately equal to the speed of the zonal wind at the upper boundary, so that they are not readily influenced by disturbances at the lower boundary. A consequence is that isolated upper-level disturbances are generally incapable of locking on to disturbances that they themselves induce at lower boundaries. Nevertheless, they can lock on to pre-existing, isolated lower-level disturbances.

Two final thoughts:

1. It is notable that a conference on mesoscale processes opens with a session on extratropical cyclones. It is true that cyclones share with fractal structures (even if they do not satisfy the definition of them) the property that the closer you look the more detail you see. Inevitably you will find structure that is undeniably mesoscale, however you choose to define it. You certainly do not have to stretch the definition out to the upper limit of meso- $\alpha$, which is much bigger than what Fujita originally had in mind. Much of this structure is different from the kind of phenomenology first inspiring the term "mesoscale," but it is interesting all the same. Many (but not all) deep cyclones have intense inner cores on rather small scales. We do not know why some do and some do not, and I did not hear that issue discussed much. Perhaps Hedley's and Yau's paper came closest to it.

2. The spotlight has been on explosive intensification of oceanic cyclones for nearly ten years now, and the research on them is showing signs of maturity. A decade ago the ability of numerical models to simulate this sort of thing was pathetic. We have seen a great increase in the capability of numerical models, both research and operational, to represent this phenomenon, and we find ourselves repeating the same kinds of things: latent heat release is important, as is strength of upper-level forcing and effective static stability. The role of surface fluxes of heat and moisture remains somewhat in dispute. There is little doubt that these fluxes are important, but is this importance exercised in advance of the cyclogenesis through conditioning of the air or is it exercised in situ? There is probably no single answer for all cases. It is important to get the grid points closer together, but that seems to be more a matter for computer logistics and for algorithmic ingenuity than for physical insight.

\section{b. Session 2. Mesoscale cyclonic vortices (Ying-Hwa Kuo, chair) \\ J. Nielsen analyzed National Meteorological Center}

(NMC) Nested-Grid Model (NGM) forecasts for two small-scale cyclones observed during the Genesis of Atlantic Lows Experiment (GALE). He found that vertical motion induced by latent heat release contributed to the generation of low-level potential vorticity at different times in the evolution of the cyclones. In both cases, the intense vertical motion over regions of conditional symmetric instability was responsible for the intensification of the small-scale cyclones in the model. He noted that the distribution of precipitation in the NGM is extremely sensitive to the initial moisture field when symmetric instability is present.

P. Alpert and Y. Shay-El calculated heat and moisture budgets over the eastern Mediterranean using the European Centre for Medium-Range Weather Forecasts (ECMWF) $2.5^{\circ}$ analysis for six winters during 1982-1987. They found that diabatic heating associated with latent heat release was considerably larger for eastern Mediterranean cyclones than for cyclones over the northern Baltic region. The surface sensible- and latent-heat fluxes from the warm Mediterranean were also important energy sources for the eastern Mediterranean cyclones.

M. Douglas, M. Shapiro, and L. Fedor presented an analysis of a polar low along the ice edge east of Greenland based on data gathered using a NOAA WP-3D research aircraft. This polar low, which occurred during 17-18 March 1989, showed several interesting features. The $950-\mathrm{mb}$ temperature analysis indicated that the warmest air $\left(\sim-3^{\circ} \mathrm{C}\right)$ was near the vortex center, with much cooler air $\left(\sim-13^{\circ} \mathrm{C}\right)$ over the ice. The polar low revealed a warm-core structure in the lower troposphere. The largest thermal gradient was found north of the center. Analysis of the $800-\mathrm{mb}$ wind and temperature fields showed rapid weakening of the polar low with height. Exhibiting the largest amplitude in the temperature and vorticity fields near the surface, this polar low was closer in structure to a Gulf of Alaska polar low than to a Norwegian polar low observed by the same authors.

P. Pokrandt and G. Tripoli studied the interaction of convection and a polar low using a nonhydrostatic mesoscale model. They initialized the polar-low vortex by specifying angular momentum surfaces that can be arbitrarily tilted with height in the lower levels and that return to their ambient orientation aloft. By changing the tilt of the momentum surfaces while holding the potential temperature structure fixed, they varied the degree of symmetric instability in the initial conditions. Using this model configuration, they studied the effects of sea-surface temperature gradient and condensation on the development of the polar low. They found that latent-heat release is crucial for the development of the polar low. Strong sea-surface temperature gradients also contribute to the develop- 
ment, but to a lesser degree. They also found that the storm-scale circulation associated with the polar low is responsible for creating symmetric instability, which leads to the occurrence of slantwise convection.

G. Stossmeister and G. Barnes analyzed aircraft observations obtained in Tropical Storm Isabel. Their analysis showed the development of a second circulation center outside the radius of maximum wind, over $80 \mathrm{~km}$ from the original center. Airborne radar observations indicate that the development of the new center is correlated with the development of strong convection in a nearby rainband. The new center is found beneath the anvil downwind from this convection and is associated with subsidence warming. Within a matter of hours, the new center becomes dominant while the original loses its identity. The process by which the new center forms is still unclear. One possible mechanism proposed by the authors is that subsidence warming may produce a sizeable hydrostatic low beneath the anvil, which may, in turn, induce a response in the rotational wind field if the pressure perturbation is maintained for a long enough period.

K. Kanak and G. Tripoli conducted three-dimensional simulations of a tropical cyclone using a nonhydrostatic model, with the objective of resolving tropical cyclone circulations and convection simultaneously. The model was initialized using a modified sounding from Kingston, Jamaica, $24 \mathrm{~h}$ prior to the landfall of Hurricane Gilbert, and run with grid sizes of 50 and 20 $\mathrm{km}$. The outcome was that proper representation of gravity-wave energy transport through the use of radiative boundary conditions, nonhydrostatic dynamics, and consideration of three dimensions are important for accurate simulation of the development and structure of a tropical cyclone.

\section{c. Session 3. Mesoscale predictability (Donald J. Perkey, chair)}

The mesoscale predictability session, a new addition to the conference, consisted of three papers. Two of these papers focused on the variation of numerical simulations resulting from "lagged" initial conditions, i.e., simulations which begin at $12 \mathrm{~h}, 24 \mathrm{~h}, 36 \mathrm{~h}, 48 \mathrm{~h}$, ..., preceding a selected verification time. The third paper addressed the predictability of precipitation distributions within a midlatitude cyclone.

From a paper by Y.-H. Kuo and S. Low-Nam, Kuo described a set of numerical simulations made using The Pennsylvania State University/National Center for Atmospheric Research (Penn State/NCAR) regional model in which they simulated the ERICA IOP4 cyclogenesis event beginning at $24 \mathrm{~h}, 48 \mathrm{~h}, 72 \mathrm{~h}$, and $96 \mathrm{~h}$ in advance of the verification time (0000 UTC 5 January 1989). Kuo emphasized the importance of using a hemispheric model domain and interactive grid nesting when doing these types of mesoscale predictability experiments. The error growth rate using these "unbounded" conditions was considerably larger than those reported by investigators who used prescribed lateral boundary conditions. He also indicated that the intensity of this storm was better simulated by the range of simulations than was its location.

$P$. Roebber described results from a somewhat similar set of experiments, but compared the results of two operational models (NMC's NGM and the Canadian Meteorological Centre's Regional Finite Element Model) for an ensemble of 42 cases. These cases ranged from ones that filled to ones which rapidly deepened during the simulation periods. He noted that there was considerable variance within a given set of "lagged" simulations. He also stated that the consistency of the simulations was not necessarily indicative of the accuracy of the simulations; i.e., that a set of simulations beginning at $48 \mathrm{~h}, 36 \mathrm{~h}, 24 \mathrm{~h}$, and $12 \mathrm{~h}$ before the verification time yields similar results does not indicate that the results are any more accurate or valid than a set of simulations that exhibit a wide degree of variation between each other.

H.-R. Cho compared the results of a two-dimensional semigeostrophic frontogenesis model initialized using a prescribed reference state with a corresponding model initialized using a perturbed version of the same reference state. His results indicate that small temperature or potential vorticity (PV) anomalies may induce significant ageostrophic flow perturbations. These perturbations may result in latent heat release, which, in turn, may feed back positively with the PV anomaly field. This feedback has the same time scale as frontogenesis, and thus indicates that the accuracy and resolution of the initial data may strongly influence the accuracy and resolution and, therefore, the predictability of the precipitation field.

\section{d. Session 4. Fronts and frontogenesis (Ronald E.}

Stewart, chair, with G. W. Kent Moore)

This session was concerned with a wide variety of features related to fronts and frontogenesis. The first four papers were observational studies of oceanic fronts, fronts over land, and sea breezes. The last eight papers were theoretical, focusing on frontal circulations, frontogenesis, and frontal instabilities.

Three of the observational papers dealt with frontal systems. N. Bond and M. Shapiro reported on a nonprecipitating front that extended from the tropopause to the surface, observed during the Ocean Storms field experiment over the North Pacific Ocean. Near the surface, the front was very sharp $(<1-\mathrm{km}$ wide zone of strong vertical motion), although associated cross-front temperature differences were only about $1^{\circ} \mathrm{C}$. This feature also exhibited a great deal of 
along-front variability and was responsible for drawing stratospheric ozone down to at least $600 \mathrm{~m}$ above the surface. G. Hakim reported on an unusual cold front that affected the east coast of the United States. Termed a "sidedoor" cold front because of its propagation to the southwest and its parent cold front, the feature exhibited characteristics resembling a density current. It is believed that differential friction and heating along the coastline were crucial in its development. P. Hobbs, J. Locatelli, and J. Martin presented a conceptual model of frontal systems that moved eastward from the Rockies during the GALE project. The conceptual model depicts a cold front aloft that sometimes can be associated with severe weather, including tornadoes. The model also illustrates that a surface trough occurs $\sim 200 \mathrm{~km}$ to the west of the front aloft, but a distinct cold front is not always present at the surface.

Along the flat coast of South Australia, a strong sea breeze commonly occurs, although it is mainly confined to the lowest $\sim 1 \mathrm{~km}$ of the atmosphere. $\mathrm{H}$. Kraus and $\mathrm{J}$. Hacker examined this feature using detailed aircraft observations. Confluence and shear both contribute to frontogenesis in the lower regions. However, the shear has a frontolytic effect at the head of the circulation and is also responsible for the generation of lobes and clefts in the middle of the circulation.

The following four theoretical papers were concerned with idealized models of frontal circulations and frontogenesis. J. Sun derived a version of the Sawyer-Eliassen equation applicable to the case in which gradient wind balance is assumed. She applied this equation in an investigation of the secondary circulations in an asymmetric jet. W. Blumen analyzed the role that dissipation has on the frontal zones that develop in a semigeostrophic Eady wave. The introduction of a thin dissipative layer allows the solutions to be extended past the time of formation of the frontal discontinuity. The magnitude of the dissipation required for equilibration of the wave was found to be in good agreement with observations. M. Montgomery and $B$. Farrell explored the effect that latent heat release has on frontogenesis associated with potential vorticity anomalies. The case in which surface development is induced by upper-level forcing was considered, as well as that in which neutral surface waves are destabilized by surface potential vorticity anomalies. For both cases, it was shown that the introduction of latent heat results in an enhancement of the frontogenetic process. G. Moore examined the effect of surface heating on the structure of atmospheric frontal zones. Surface heating was shown to induce a low-level potential vorticity anomaly that acts to increase the baroclinicity and reduce the static stability associated with a front. In doing so, it leads to an increase in the rate at which waves can develop along the front.

The concluding four papers dealt with frontal instability and three-dimensional aspects of frontogenesis. S. Malardel and A. Joly reviewed theories that have been proposed to explain the growth of frontal waves. Emphasis was placed on the role that potential vorticity anomalies have on the development of these waves. In particular, they demonstrated that when such an anomaly is found in the lower troposphere, a frontal instability with a length scale $\sim 1000 \mathrm{~km}$ is present. A. Joly and A. Thorpe considered the stability of a front in a growing baroclinic wave. By use of the so-called linear tangent method, they were able to relax the conventional time-scale separation assumption. In applying this new formalism, they were able to show that shearing deformation can act to increase the growth of frontal waves. F. Lalaurette compared the life cycle of a baroclinic wave in a primitive equations model with that of earlier work of Hoskins and coworkers in which the geostrophic momentum approximation was made to form the semigeostrophic equations. Unlike the earlier semigeostrophic models, but in agreement with other primitive equations models, a strong "bent-back" warm front developed. Such differences in frontal structure were attributed to differences in the ageostrophic flow patterns in the semigeostrophic and primitive equations for finite-amplitude disturbances, where Rossby numbers are no longer much less than unity. $\mathrm{H}$. Davies, $\mathrm{H}$. Wernli, and C. Schär investigated the influence that ambient barotropic shear has on frontogenesis within a growing semigeostrophic baroclinic wave. The introduction of barotropic shear was shown to break the symmetrical "high-low" pattern in the evolving wave and to exert a strong influence on the structure of cold and warm fronts.

\section{e. Session 5. Banded precipitation features in fronts and cyclones: I (Mohan K. Ramamurthy, chair)}

The first session on banded precipitation features in fronts and cyclones featured a total of seven papers, all of which addressed the problem from an observational standpoint. A notable aspect of these papers was the diversity of locations where these precipitation bands and associated structures were observed. The phenomena and locations ranged from marine rainbands over the Atlantic during GALE to subsynoptic structures over Finland, and from precipitation bands in the near-freezing region over Canada to upslope snowbands in Colorado.

The session started with a talk by M. Powell on the structure of the marine boundary layer associated with 
rainbands located $\sim 500 \mathrm{~km}$ off the Florida/Georgia border during a rainband-boundary-layer experiment. Using a variety of observing platforms, he documented the mesoscale kinematic structure, as well as the detailed boundary-layer characteristics, within and adjacent to a narrow line of convective cells. These convective cells, with vertical extension of 6 to $9 \mathrm{~km}$ and updrafts of 5 to $10 \mathrm{~m} \mathrm{~s}^{-1}$, were found along a stationary front, which also had characteristics of a warm front. The convective cells coincided with a region of strong gradient of $\theta_{\mathrm{e}}$ in the direction normal to the band and were situated in a region of synopticscale convergence.

P. Saarikivi and T. Puhakka reported on their study of subsynoptic structures and processes in a decaying cyclone using high temporal resolution ( $<1 \mathrm{~h}$ ) radiosonde observations during a frontal passage. Their analysis, performed and interpreted in the context of classical warm and cold conveyor belt flows, showed that the mesoscale structure of the occlusion was characterized by a warm frontal zone and, lying above it, a series of circulations, often described in the literature as warm tongues and cold surges, parallel to the front. They found that the observed wavelength of these circulations and the associated thermal and kinematic features agreed well with the theory of conditional symmetric instability (CSI). Despite this agreement, only small areas of CSI were observed, the most significant of which was found behind the surface front in the occlusion. The formation of the moist symmetrically unstable region was attributed to advection of cool, dry, midtropospheric air above the moist surface frontal air.

The next paper, by M. Shields, R. Rauber, and M. Ramamurthy, dealt with mesoscale organization of precipitation bands in a Midwest winter cyclonic storm. These bands were observed during a field experiment conducted by the investigators at the University of Illinois. Three distinct types of mesoscale precipitation bands were identified, with vastly different orientations, directions of movement, and mechanisms of development. The first band, a very narrow feature that lasted over $5 \mathrm{~h}$ and remained stationary with respect to the warm front, was attributed to hydrostatic stationary gravity waves along the frontal surface. The second band was identified with a convergence zone accompanying the inverted trough at the leading edge of the arctic air. The final group of bands, observed late in the storm, was oriented parallel to the thermal wind vector in the middle troposphere and situated in a neutrally stratified atmosphere with respect to CSI.

J. Marwitz reported on a case study of a heavy snowfall event in Oklahoma on 14 December 1987. On the following day, this storm was associated with a very large amplitude gravity-wave event and one of the most intense continental cyclones ever to be observed. He showed single-Doppler analysis of National Severe Storms Laboratory (NSSL) radar data and emphasized the importance of frontogenetical forcing mechanisms in the formation of the band.

R. Rassmussen, A. Crook, and C. Kessinger showed dramatic footage of the CP-2 radar display during the 15 November 1987 snowstorm along the Colorado Front Range that contributed to the crash of Continental Airlines flight 1713 due to severe icing. The snowband that led to severe icing initially propagated from the southeast to the northwest (against the low-level flow) and then suddenly became nearly stationary over Stapleton Airport in Denver. The authors simulated the precipitation bands using the Clark nonhydrostatic cloud model, showing that the bands were initiated by the passage of a secondary cold front from the north. Their initial results, using a slightly stable sounding, showed bands in the model propagating opposite to the direction observed in the real atmosphere. However, another integration using a slightly unstable sounding resulted in model band movement very similar to that observed. This extreme sensitivity to the thermodynamic profile was attributed to the deeper convection in the latter simulation being steered by the mean flow, as opposed to a shallower feature that was advected by the low-level winds in the first simulation.

G. Raga, R. Stewart, and N. Donaldson discussed the structure of the precipitation type transition region near $0^{\circ} \mathrm{C}$ in a winter storm. The observations came from the 1986 Canadian Atlantic Storms Program (CASP) field project, during which a transition zone was sampled using in situ aircraft, radar, rawinsonde, and surface observations. The primary conclusion from their study was that the mesoscale circulation near the melting region resembled that of the synopticscale conveyor belts in midlatitude cyclones, except on a much smaller scale.

The final presentation of the afternoon was given by A. Thorpe and S. Clough on the potential vorticity (PV) structure of cold fronts observed in the FRONTS 87 field project. Their high-resolution observations revealed several ubiquitous features of the PV distribution at cold frontal boundaries, such as negative PV in the upper troposphere on the anticyclonic side of the jet and negative PV in the midtroposphere on the cold side of the frontal zone. The latter feature is associated with buckled constant absolute momentum surfaces and is coupled with a positive PV anomaly straddling the surface front. They also raised a new issue concerning the dynamic role of evaporation of snow within and beneath the frontal surface and the resulting cooling region underneath the ascent region (where heating occurs due to condensation), leading to a 
dipole forcing of the PV distribution. Such a dipole forcing will lead to a complex mesoscale structure in the local circulation, for which they showed some observational evidence.

\section{f. Poster Session P1. Extratropical cyclones and fronts (David J. Knight, chair)}

Fifteen posters were presented in this session, which included a healthy balance of observation, theory, and numerical simulation. The first three posters were devoted to upper-level fronts. These fronts generally form upstream of geopotential troughs and are forced primarily by tilting and confluence. Using isentropic analysis, F. Sanders, L. Bosart, and C.-C. Lai documented the formation and development of an observed upper-level front. The front formed at about $300 \mathrm{mb}$, then descended toward $850 \mathrm{mb}$ at a rate of about $5 \mathrm{~cm} \mathrm{~s}^{-1}$. They argued that this front was forced by confluence of the ageostrophic winds associated with gradient wind balance in a ridge-trough system, resulting in an indirect circulation at the entrance of the frontal region and tilting of the isentropes. An analysis of potential vorticity (PV) on isentropic surfaces revealed that on the warm side of the front $P V$ is conserved and stratospheric air descends to tropospheric levels. However, on the cold side of the front, PV was observed to increase significantly at low levels. This increase could not be explained entirely by observational uncertainty, thus suggesting the influence of diabatic and frictional processes. C. Konor, C. Mechoso, and A. Arakawa used an isentropic model on a $\beta$-plane to simulate upper-level frontogenesis. Far upstream of the trough, they found that confluence is the primary frontogenesis mechanism. Tilting by the transverse ageostrophic circulation (centered on the warm side of the front) dominates frontogenesis in the rest of the front. K. Hines and C. Mechoso also performed numerical simulations of upper-level frontogenesis. They found considerable differences between simulations on a sphere and on a $\beta$-plane. Initial conditions had to be especially designed to produce a realistic simulation of an upper-level front on a sphere. They found that tilting by the vertical motion forced by divergence of the across-jet ageostrophic wind is the dominant frontogenesis mechanism. All three studies suggest that upper-level frontogenesis is due primarily to variations in the motions across the front and so may be thought of as a two-dimensional process. Nevertheless, establishment of the forcing mechanisms for the indirect across-front ageostrophic circulation requires the three-dimensional structure of the baroclinic wave.

Four posters dealt with the mesoscale structure and instabilities of low-level fronts. T. Puhakka and P. Saarikivi diagnosed vorticity production associated with "cold surges" in an occlusion. The cold surges were associated with enhanced rainfall at the ground. Vertical vorticity was due primarily to convergence and tilting associated with the enhanced updrafts in the cold surges. H.-M. Juang presented numerical simulations of an observed cold front with mesoscale along-front variation in vorticity and frontal strength. Deformation and convergence acted to produce the regions of enhanced temperature gradient. Convergence resulted in enhanced vorticity production in several zones along the front. However, this was overwhelmed by negative vorticity production by the solenoidal term (in a terrain-following sigma coordinate system) just downwind of the Rockies, where strong frontogenesis was occurring. The terrain-induced surface pressure distribution combined with increasing temperature gradients tended to enhance the negative solenoidal forcing, diminishing the vorticity along the portion of the front adjacent to the mountains. J. Clark and T. Salem used the Hoskins-Bretherton zero potential vorticity model to investigate mesoscale frontal instabilities. Prescribed deformation leads to decreases in the horizontal extent and increases in the strength of the unstable temperature gradient. This results in a smaller-scale and less sharply defined shortwave cutoff relative to cases with broad temperature gradients. W.-Y. Sun considered baroclinic instability in the presence of vertically varying static stability. He found that both longwave (Eady type) and shortwave disturbances can grow. The short waves develop in the lower atmosphere where the static stability is less and grow faster as the stability aloft is increased.

Using natural coordinates, G. Bell and D. Keyser considered the conversion from shear to curvature PV during the formation of a $500-\mathrm{mb}$ cutoff cyclone observed during GALE. They found that parcels upstream of the trough had considerable shear PV. As the parcels approached the trough axis, the shear PV was converted to curvature PV, while parcels leaving the trough lost curvature PV and gained shear PV. Consideration of conversions between shear and curvature PV was shown to be useful in explaining the changing phase between jets and the parent baroclinic wave during the evolution of the latter. R. Rotunno and $D$. Keyser reconsidered the turbulent mixing of PV, a topic of some recent controversy. The central issue was whether $\mathrm{PV}$ is mixed downgradient by turbulence or if heat and momentum are mixed downgradient. The outcome was shown to depend on how one chooses to define Reynolds-averaged PV. For conventional averaging, where mean PV is understood to be the scalar product of averaged vorticity and averaged potential temperature gradient, PV "mixing" is controlled by the turbulent transports of heat and 
momentum. It is also possible to define the mean PV as the average of the scalar product of vorticity and potential temperature gradient. In this case, it is necessary to parameterize the eddy flux of PV; arguments for and against downgradient mixing of $\mathrm{PV}$ were proposed, without conclusive resolution. J. Keller examined the role of synoptic-scale forcing in the reduction of the Richardson number $(\mathrm{Ri})$, and the subsequent formation of clear-air turbulence (CAT). $\mathrm{He}$ found good agreement between the tendency diagnosed from the prognostic equation for $\mathrm{Ri}$ and the observed tendency, suggesting the possibility of accurately forecasting the occurrence of CAT events and the associated energy dissipation.

Coastal cyclogenesis observed during ERICA and GALE was the subject of three posters. E. Donall, M. Shapiro, and P. Neiman looked at the role of varying model physics in the simulation of the rapidly intensifying marine cyclone during ERICA IOP-4. The model did a reasonable job of reproducing observed cyclone and frontal structure. It is apparent that surface fluxes of heat and moisture as well as latent heating are crucial to the accurate simulation of oceanic cyclones. F. Robertson and D. Perkey compared model-derived cloud and precipitation structures with satellite microwave measurements for the same ERICA cyclone considered in the previous presentation. Reasonable agreement between model-simulated and observed moisture structure points toward the usefulness of satellite data in verifying model simulations and their possible role in model initialization. T. Trunk and $\mathrm{L}$. Bosart analyzed a subsynoptic-scale cyclogenesis event observed during GALE. The study emphasized the interaction of an upper-level disturbance with a pre-existing frontal zone along the edge of the Gulf Stream. The cyclone intensified rapidly when convection broke out near the cyclone center. Differential diabatic heating between land and water played a role in forcing frontogenesis ahead of the storm, preconditioning the environment for additional convection. These studies point out the complicated interactions between upper-level disturbances, lower-level baroclinicity, surface heat and moisture fluxes, and latent heating that occur during rapid oceanic cyclogenesis.

S.-H. Chou and T. Miller considered the role of moisture and convective parameterizations on the development of baroclinic waves with an embedded region of conditional symmetric instability. They found that moist processes increase the growth rates of the waves. Adoption of a convective parameterization virtually eliminated the symmetric instability. Different convective parameterizations produced widely varying results, indicating the difficulties and uncertainties associated with such parameterizations. W. Hao and D. Keyser used a hierarchy of approximations to determine the vertical circulation in an idealized baroclinic wave, given the mass field (geostrophic flow). The quasi-geostrophic system overestimated the amplitude, but did a reasonable job of diagnosing the phase of the vertical circulations. Using the geostrophic momentum and balanced approximations brought the diagnosed circulations progressively closer to the model simulation. The success of the various approximations in reproducing the vertical circulation (the divergent flow) was qualified with the caution that poorer overall agreement was found in diagnoses of the nondivergent ageostrophic circulation.

\section{g. Poster Session P2. Mesoscale convective systems} and mesoscale vortices (Mark R. Hjelmfelt, chair)

This session consisted of eight presentations relating mesoscale systems to their environment. The first four employed numerical computer simulations. T. Sasamori and Y. Ogura used a numerical cloud model to illustrate the importance of the sloping terrain across the Great Plains coupled with low-level moisture from the south and upper-level westerly winds for the development of large convective storms and severe weather. The second presentation, by K. Waight and J. Zack, discussed simulations with the Mesoscale Atmospheric Simulation System (MASS) three-dimensional mesoscale model of a case from 28 June 1986 that was documented during the Cooperative Huntsville Meteorological Experiment (COHMEX). Their results underscored the importance of mesoscale moisture information in initializing mesoscale prediction models, and further illustrated the continuing difficulty in precise simulation of the details of timing and location of ordinary warm-season convective storms, even when mesoscale data are included. J. Song and M. Kaplan examined three-dimensional modeling of thunderstorms on a much smaller scale using data from the same day in COHMEX and illustrated the value of detailed initialization using mesonet winds. A threedimensional high-resolution cloud model was found to give good comparisons with observed storm development with initialization using a detailed mesoscale surface wind field from the Portable Automated Mesonet (PAM) network that was available during COHMEX. The fourth presentation, by C. Seman, examined convective symmetric instability and illustrated the importance of baroclinicity in enhancing the development of deep convection.

M.-S. Lin and C.-C. Yang presented observations showing that there are strong similarities in many features of mesoscale convective systems off the coast of Taiwan relative to those observed in the continental United States. This emphasizes the potential transferability of results of studies of continental midlatitude MCSs to other parts of the world. A poster 
by $Y$. Miura discussed observed structural characteristics of mesoscale vortices on the scale of 70 to 150 $\mathrm{km}$ associated with cold-air outbreaks over Japan.

The last two presentations considered various aspects of tropical cyclones. E. McCaul discussed spatial and temporal patterns in tornadogenesis within landfalling hurricanes. He also showed some results on the preferred time of day of landfall, relating landfall times to the temporal distribution of hurricane translation speeds that he attributed to tidal oscillations of the atmosphere. F. Marks and P. Black presented dramatic observations from a NOAA WP-3D research aircraft of a very intense, small-scale $(\sim 1 \mathrm{~km})$ vortex located just within the eyewall of Hurricane Hugo.

\section{h. Poster Session P3. Thermally and mechanically forced flows (Hampton N. Shirer, chair)}

Three presentations dealt with characteristics of the sea breeze. J. Intrieri and L. Olivier detailed a complete diurnal cycle of a sea-breeze circulation that was observed during the Land/Sea Breeze Experiment (LASBEX) in September 1987. Doppler lidar rangeheight indicator $(\mathrm{RHI})$ measurements of the radial wind field, together with measurements from other sensors, were used to depict the onset, peak, dissipation, and interaction of the sea-breeze and landbreeze flows. D. Jacob, W. Koch, L. Levkov, and D. Eppel used a three-dimensional, nonhydrostatic, anelastic Boussinesq mesoscale model to investigate the effects that cloud formations have on the characteristics of sea-breeze circulations over a peninsula. They presented a simulation of an observed seabreeze event over northern Germany and compared the results with observations. They showed that differential heating induced two distinct sea-breeze fronts along the coasts of the North and Baltic Seas, and that these two fronts merged over Jutland to form a single updraft cell, as was observed on an Advanced VeryHigh-Resolution Radiometer (AVHRR) satellite image. S. Zhong, J. Leone, and E. Takle used both twodimensional and three-dimensional, anelastic, hydrostatic, finite-element models to study the low-level wind flow that is driven by the surface heating patterns over the Kennedy Space Center/Cape Canaveral area of Florida. A simulation of a typical day sampled during the Fall 1988 intensive data-collection period of the Kennedy Space Center Atmospheric Boundary Layer Experiment (KABLE) was presented. They demonstrated that cooling of the boundary layer by the Indian River created a river breeze that substantially influenced the sea-breeze circulation, in good agreement with observations.

Two presentations explored coastal wind characteristics. M. Abreu and P. Bannon compared solutions to semigeostrophic $f$ - and $\beta$-plane models to study western coastal flows driven by land-sea temperature contrasts. They used these models to investigate why the low-level winds along the Pacific coasts of North and South America are generally directed equatorward near the surface. The $\beta$-plane model successfully represented the typically observed surface flow, flow reversal aloft, stable stratification, and subsidence in the region. J. Reisner and E. Takle showed one- and two-dimensional numerical simulations of thermally and mechanically forced ageostrophic coastal jets; such jets were measured near the ocean during the Coastal Ocean Dynamics Experiment (CODE), which was held off the northern coast of California. With a two-dimensional, finite-element, hydrostatic, incompressible model, they demonstrated that large horizontal pressure gradients can develop that support measured wind speeds as high as $32 \mathrm{~m}$ $\mathrm{s}^{-1}$ at $200 \mathrm{~m}$. These gradients were created by a combination of strong inland heating, an upwellingcooled marine layer, coastal mountains, and a sloped inversion.

Three presentations discussed aspects of boundary-layer modifications by lakes. R. Arritt showed observations that were obtained using a tethered-balloon sounding system of the profiles of wind, temperature, and humidity measured near a small lake in eastern Kansas. Two case studies were presented, the first of a typical clear summer day in June 1989, and the second of a record-setting cold-air outbreak in August 1989. Both studies demonstrated that significant alterations of the boundary-layer structure can occur in the vicinity of lakes. P. Cehelsky, M. Ablowitz and $S$. Ko presented an observationally based analysis for determining the characteristic spatial and temporal scales of lake-effect snowstorms. The physical effects considered were an adverse vertical temperature gradient, a vertically shearing horizontal wind, rotation about a vertical axis, and dissipation. The obtained scales were used to explain the observed alignments and aspect ratios of the intense snowbands that develop downwind of Lake Ontario. R. Reinking, R. Kropfli, and B. Martner showed some preliminary results from the Lake Ontario Winter Storms (LOWS) experiment that was held during January and February 1990. They presented an overview of the LOWS experiment as well as several lake-effect storm observations that were obtained using a NOAA WP$3 D$ research aircraft and a triangular array of instruments that included wind profilers, microwave radiometers, a radio acoustic sounding system, and an Xband Doppler radar. Two detailed sets of results were shown, the first of a lake-effect snowstorm on 12 January 1990, and the second of a freezing rain event on 15 February 1990.

Other mesoscale responses to surface forcing were 
investigated in the remaining two presentations. J. Fast studied thermally induced mesoscale circulations that were produced in the central United States by gradients in soil type, soil moisture, vegetation, snow cover, and cloud cover. An existing hydrostatic, anelastic, three-dimensional, coupled earth-atmosphere mesoscale numerical model was modified to represent the effects of soil moisture and vegetation distributions. Observed surface and upper-air data were used to initialize the model, and it was shown that horizontal inhomogeneities in soil moisture significantly affect the temperature and moisture structure of the simulated boundary layer. W.-Y. Sun and C.-C. Wu used a two-dimensional mesoscale model to study the diurnal variation of the fair weather dryline. Owing to daytime convection, they found that the dryline moves eastward, while owing to nighttime surface cooling it moves westward. The horizontal soil moisture gradient, the sloping terrain, and the low-level vertical wind shear across the dryline were demonstrated to play crucial roles in the formation and maintenance of the strong moisture gradient across the dryline.

\section{i. Session 6. Banded precipitation features in fronts and cyclones: II (Alan J. Thorpe, chair)}

The common theme that linked the papers in this session was the implication in theoretical or numerical models of the presence of conditional symmetric instability (CSI). The notion of this instability as a feature of fronts has a long history dating back to early work by Solberg, Kleinschmidt, and Sawyer, but the field went through a revival after new linear normal mode calculations were made in the late 1970 s by Bennetts and Hoskins. It is fair to say that the idea that frontal rainbands may be a manifestation of CSI has fired the imagination of theoreticians before adequate observational evidence has been amassed to verify its existence at fronts. Of course, that observational evidence, in common with that for other mesoscale structures, is difficult to obtain due to the small horizontal scale of the phenomenon. Recent observational programmes such as FRONTS 87 and ERICA are beginning to provide this evidence, but it is unclear whether there is, as yet, unambiguous verification of the existence of CSI at fronts.

The session began with a paper by J. Lafore, J. Redelsperger, and $\mathrm{P}$. Benard on a nonhydrostatic simulation of moist frontogenesis. The two-dimensional Eady problem was used as a framework for a simulation with moist and surface processes and with a sophisticated turbulence scheme. Both narrow and wide cold frontal rainbands were produced, and attention was drawn to the development of a region of high equivalent potential vorticity in the warm air that was advected toward and up the cold front. This region was produced predominantly by surface fluxes of moisture. A three-dimensional version of a similar simulation was described by D.-L. Zhang and H.-R. Cho using the hydrostatic Penn State/NCAR model. The sensitivity of the precipitation bands to the inclusion of an ice microphysical scheme was described. The results seemed to suggest a strong sensitivity to the cold clouds, with more bands being produced if ice physics are included. The authors suggested that CSI was playing an important role.

There were three papers on numerical simulations of CSI itself in the absence of frontogenesis. O. Persson and T. Warner described the nonlinear development of a CSI roll in a version of the Penn State/NCAR model. In contrast to those from other authors, the results seemed to suggest slantwise ascent oriented parallel to the absolute momentum surfaces rather than parallel to the saturated isentropes. T. Miller and S.-H. Chou described simulations from a nonhydrostatic model of a flow that had a lower layer unstable to convection, a middle layer unstable to symmetric overturning, and an upper stable layer. The results, in the form of a color video, revealed how the convection develops rapidly and ultimately forces the $\mathrm{SI}$ to break out in the middle layer. This situation may well form the basis of a good model of fronts in which often the convective activity is confined to the lower troposphere. M. Holt discussed a new approach to SI in which weakly unstable and stable flows are impulsively and continuously forced locally with anomalies of inertia and/or heat. This is the initial-value problem for symmetric instability. A parcel model, which underestimated the eddy kinetic energy, a traditional numerical model, and the so-called geometric model were applied to the problem. Zero potential vorticity lens structures observed in the FRONTS 87 project were reproduced in the numerical models.

With spectacular bravado, S. Jascourt delayed the audience from reaching the coffee queues on time by describing a model of fully nonlinear slantwise motions in two-dimensional flows. The advocacy of the Lagrangian model he presented seemed to be at odds with the previous results of Holt on the inaccuracy of the parcel method for SI. As often happens in science, an idea will occur to more than one author concurrently. Such was the case with an idea presented by $Q$. $\mathrm{Xu}$, who suggested that some rainbands may be produced by the prior existence of potential vorticity anomalies. These act to focus the development of the bands. A similar paper was presented in Session 3 by $\mathrm{H}$.-R. Cho. It then becomes an interesting question of what determines the lower-tropospheric spectrum of potential vorticity anomalies and whether routine observations have the resolution to describe these adequately.

An impression this session chair was left with is that the diagnosis of tropospheric distributions of potential 
vorticity and equivalent potential vorticity from observations is extremely important if some of the dynamical issues being raised about fronts are to be answered. Analyses of isentropic distributions of potential vorticity are recognized as important for synoptic systems, but for mesoscale weather systems where diabatic and frictional processes are important, the isentropic view is certainly not adequate. Full fourdimensional descriptions are needed to properly describe the essential dynamics of fronts and cyclones.

\section{j. Session 7. Gravity waves, convective lines, mesoscale instabilities and turbulence (Steven E. Koch, chair)}

The presentations in this session dealt with gravity waves, solitary waves, the interaction between deep gravity waves and convection, gravity currents, various mesoscale instabilities associated with jet-related wind shears, and the nature of turbulence (notably near the tropopause). There appeared to be a strong emphasis on observational and theoretical/numerical research, with only minor use of laboratory results. The bulk of the studies concerned gravity/solitary waves, including some fascinating new information about their vertical structure obtained from recent wind profiler data.

The first two papers examined the relationship between gravity waves and precipitation bands within extratropical cyclones. R. Schneider showed several very large amplitude waves (surface pressure falls of as much as $11 \mathrm{mb}$ in $15 \mathrm{~min}$ ) of long duration, accompanied by heavy convective snowbursts and surface winds in excess of $30 \mathrm{~m} \mathrm{~s}^{-1}$. One of the wave disturbances, which displayed the character of a solitary wave of depression, was characterized by a sea-level pressure minimum lower than that of the cyclone center. M. Ramamurthy, B. Collins, R. Rauber, and P. Kennedy utilized a $10-\mathrm{cm}$ Doppler radar, a $50 \mathrm{MHz}$ wind profiler, barograph, and special thermodynamic sounding data collected during a field experiment conducted near Champaign, Illinois, in 1989. The barograph data suggested the appearance of a solitary wave of depression, albeit less intense than the spectacular wave discussed by Schneider. Profiler data indicated wave trapping. The data also showed that air parcels influenced by the passage of this wave experienced vertical displacements of at least $4 \mathrm{~km}$ in less than an hour, or an upward motion in excess of 1 $\mathrm{m} \mathrm{s}^{-1}$. It was suggested that a critical level/temperature inversion acted as a nodal surface for vertical wave propagation.

F. Ralph, M. Crochet, and S. Venkateswaran discussed the vertical structure and vertical motion within a 90-min period gravity-wave event associated with a squall line. This wave train was detected in $50 \mathrm{MHz}$ profiler data collected during the FRONTS 84 field experiment in France, which revealed $20-50 \mathrm{~cm} \mathrm{~s}^{-1}$ vertical motions associated with the wave event and a structure suggestive of a wave duct resulting from the presence of a critical level.

Individually, and as a group, the preceding three papers show that mesoscale gravity-wave phenomena may occupy a substantial depth of the troposphere and are capable of exerting strong control on the weather. Given increasing evidence from recent case studies that the origin of these disturbances is related to the geostrophic adjustment process arising from mass-momentum imbalances in jet streaks, there is a great need to understand better the basic process by which gravity-inertia waves are generated by such dynamical imbalances. Furthermore, theoreticians should begin to address why strong solitary waves of depression are formed in some instances and gravitywave trains are generated in others.

Several papers dealt with the interactions between gravity waves and deep convection. Using special data collected during the Convection Initiation and Downburst Experiment (CINDE), M. Hjelmfelt presented evidence that the motion of a narrow convective rainband occurring during conditions of shallow, stable upslope flow in Colorado could be explained in terms of the propagation of gravity waves induced by the downdraft in the stable boundary layer. A large set of lightning observations and two-dimensional mesoscale model runs made in Florida sea-breeze situations by W. Lyons, D. Moon, C. Keen, and T. Nelson suggested that weak convection will tend to form first along the upwind sea-breeze convergence zone, move rapidly as some kind of "impulse" across the peninsula, and invigorate suppressed convection along the opposite coast upon its approach. Lyons hypothesized that convective development within the downwind sea breeze is retarded by the disruption of surface-based thermals due to advection by the prevailing flow over the undercutting sea-breeze layer. Deep gravity waves generated by decaying convection were simulated in a two-dimensional model by $M$. Nicholls, W. Cotton, and R. Pielke. It was shown that these oppositely propagating waves are predominantly linear in character, unlike the solitary wave disturbance generated by convective heating in the study published by Lin and Goff. It is interesting to speculate whether there may be some relationship between the "impulse" discussed by Lyons and colleagues and these convectively generated wave disturbances.

Several theoretical studies of solitary waves and gravity currents were presented. L. Kamp and R. Miesen extended the work of Grimshaw and of Gear 
and Grimshaw to study the dynamics of deep solitary waves for a fully compressible fluid in the presence of stability and shear conditions representative of the nocturnal inversion. Their research indicated that compressibility effects cannot be ignored whenever the depth of the inversion is a significant fraction of the scale height. The ability of a critical level to reflect solitary waves was examined by E. Skyllingstad using a two-dimensional nonhydrostatic model. It was found that over-reflection occurred when the Richardson number decreased at the critical level to a value of 0.25 and the stability above that level was sufficiently strong. J. Klemp, R. Rotunno, and W. Skamarock suggested a modification to the classical formula of Benjamin often used by researchers to calculate the speed of a gravity current. Rederivation of Benjamin's formula with a more realistic flow profile downstream of the gravity current suggested that its speed of propagation is not sensitive to the details of mixing in the current head. Constraints on two-layer shallowwater solutions imposed by the depth of the lower layer and the relative depth of the gravity current were also investigated. H.-Y. Chun and Y.-L. Lin studied the response of a stratified shear flow to a local heat sink using a two-dimensional nonhydrostatic model. Their model results showed that under conditions of strong low-level wind shear the resulting gravity current cannot propagate upstream, since the cold pool acts like an obstacle. Furthermore, vertical propagation of wave energy is prevented by the trapping effect of a critical level if its height is suitably chosen, resulting in resonant enhancement of wave amplitude.

Mesoscale phenomena appearing on satellite imagery as a group of propagating cloud wavelets in the vicinity of a strong subtropical jet stream were the subject of a theoretical investigation by $D$. Stevens and P. Ciesielski. The earlier suggestion by Ciesielski et al. that these $300-400 \mathrm{~km}$ wavelength disturbances were a manifestation of inertial instability was called into question by the more recent linear model results that show a new mode with larger growth rates, which owes its existence to vertical shear associated with the jet.

The final two papers of this session were concerned with the topic of turbulence. R. B. Smith and E. Salathé showed aircraft observations revealing a step-like increase in the amplitude of small-scale temperature fluctuations at the tropopause, a tendency for power spectral slopes to be near $S=-2$ in the stratosphere, and a positive correspondence between fluctuations in temperature and vertical velocity in the troposphere. These analyses led to some discussion about the role of gravity waves and diffusion in producing these structures. The topic of "turbulence collapse," in which part of the energy of three-dimensional turbulence is transferred into two-dimensional turbulence following removal of the original source of the turbulence in the presence of strong stratification, was discussed by $D$. Etling. He speculated that the collapse of protruding thunderstorm updrafts at tropopause level could be a candidate source for such turbulence with subsequent upscale energy transfer, although it is uncertain that the wake-like cloud features seen in satellite imagery could be explained as being manifestations of just such a process.

\section{k. Session 8. STORM meeting review (Richard A. Dirks, chair)}

This session comprises five presentations that provided a broad overview of the current activities of the National STORM (Stormscale Operational and Research Meteorology) Program and the more focused planning for the STORM I (central United States) multi-scale field experiments and mesoscale four-dimensional data assimilation.

$R$. Dirks began with a report on the ongoing activities to develop an interagency mesoscale initiative for fiscal year 1992 under the Federal Subcommittee on Atmospheric Research (SAR) of the Committee on Earth Sciences (CES). Preparation of this initiative is following the same process that was used successfully by the CES to enact the Global Change Program. The initiative, entitled the U.S. Weather Research Program, draws heavily from the STORM Program plans. Nine federal agencies are participating in the planning of the initiative on a working team chaired by W. Hooke of the National Oceanic and Atmospheric Administration (NOAA).

Next, D. Johnson described the objectives of the STORM I Winter Phase Field Experiment. The primary goal is to improve the understanding and prediction of winter storms and associated precipitation over the central U.S. Core objectives are: i) to describe the structure and organization of precipitation regions associated with cyclones, jet streaks, and fronts; ii) to understand the dynamics and physics of precipitation regions associated with cyclones, jet streaks, and fronts, with emphasis on interactions across a broad range of scales; and iii) to assess the predictability of winter storms and improve forecasts of the timing, location, quantity, and type of precipitation. Current plans call for this experiment to be conducted during Jan.-Apr.1994, with a limited systems test/frontal study to be held during Feb.-March 1992.

The goals of the STORM I Spring/Summer Phase Field Experiment, presented by W. Cotton, are quite similar to those for the Winter Phase, except that the focus is on mesoscale convective systems. Current plans call for this experiment to be conducted during April-July 1993. 
J. Cunning presented a brief overview of the experimental design for these field experiments. The STORM I domain extends from the Rocky Mountains eastward to the Mississippi Valley and from central Texas northward to the Dakotas. Within this area there will be a smaller meso- $\beta$-scale observational array and a nested meso- $\gamma$-scale array.

The final speaker, Y.-H. Kuo, described the fourdimensional data assimilation (4DDA) and research numerical weather prediction activities that are planned as part of STORM and STORM I. Kuo pointed out that plans call for real-time 4DDA and research numerical weather prediction to be carried out at several locations during the STORM I field experiments. This should allow investigators to begin to assess the utility of mesoscale numerical prediction models in support of field projects.

\section{Session 9. Squall lines: Observations and models}

(Richard Rotunno, chair)

M. Chong presented an observational determination of the heating and moistening of the atmosphere by a squall line off the West African coast. He discussed similarities and differences with previous such studies. S. Trier and D. Parsons discussed their simulation of a heavily precipitating convective line observed near Taiwan. The good qualitative agreement of the simulation with the data suggests that the local vertical wind shear and latent instability are primary influences. E. Rasmussen and S. Rutledge presented an analysis of the horizontal vorticity in an evolving squall line observed in Australia. They found the systematic changes in the vorticity to be broadly consistent with simple models of the temporal evolution of squall lines.

J. Cram presented results from a hydrostatic mesoscale model concerning the initial development of a pre-frontal squall line. The simulations suggest that the initial line of convection forms at the surface cold front, but then re-forms further away due to frontolytical effect of the convection. A. Tremblay presented simulations of a larger-scale cyclone (order $500 \mathrm{~km}$ ) with a nonhydrostatic convection model. He found a strong generation of gravity waves near the cyclone center, the formation of a squall-line-like feature ahead of the surface cold front, and an enhancement of the growth rate and strength of the cyclone when the effects of moisture were included.

S. Garner and A. Thorpe used a convection model with simplified thermodynamics and water processes to study the effects of shear on squall lines. In their model, the effect of shear is to allow the convection to move with the cold pool and hence achieve a longlived state. R. Fovell reported on simulations with a nonhydrostatic cloud model that included the Coriolis effect. He found that the Coriolis effect keeps the subsidence-warming (due to the convection) near the convection and so leads to the decay of the squall line.

\section{m. Joint Session J1. Mesoscale phenomena and processes influenced by topography: I (Dale $R$. Durran, chair)}

This session featured observational, theoretical, and numerical modeling results. $S$. Koch, $P$. Dorian, and $P$. Kocin described the evolution of a strong surface cold front, its relationship to ageostrophic circulations associated with a jet streak, the orographic influences on the front, and the evolution of the front into a structure similar to a density current. R. K. Smith presented observations of the New Zealand Southerly Change, noting how foehn effects appear to enhance the temperature gradients associated with this orographically influenced cold front. S. Young and D. Knight discussed numerical simulations of cold-air damming resulting from geostrophically balanced flow across a two-dimensional mountain range. Their simulations showed a nonlinear feedback acting to increase the horizontal temperature gradients and the degree of blocking in the cross-mountain flow. They hypothesized that this mechanism may be responsible for frontogenesis east of the Appalachian Mountains. Additional two-dimensional simulations of cold fronts interacting with topography by K. Keuler, J. Kerkmann, H. Kraus, and E. Schaller revealed that the propagation speed of cold fronts was greater in cases where the front traversed a mountain than in cases with flat topography.

M. Fritsch, J. Kapolka, and P. Hirschberg discussed observations suggesting that the combined effects of differential radiative heating and evaporative cooling from clouds and precipitation contribute to the development of the cold pool in cold-air damming events. J. Tilley and P. Webster described numerical simulations suggesting that East Asian cold surges are dynamically similar to trapped edge waves consisting of a combination of baroclinic Kelvin modes and barotropic shelf modes.

M. Kaplan and V. Karyampudi presented results from a complex numerical simulation of flow over the Rockies and suggested that the downslope winds and the momentum fluxes in the associated gravity waves influenced secondary circulations and severe weather well downstream from the mountains. A. Marroquin and J. Brown described an NGM (nested grid model) forecast that underpredicted the amplitude of an inverted trough that developed downstream of the Rocky Mountains. They noted that an identical adiabatic simulation, with convection and radiation parameterizations suppressed, did a much better job of forecasting the development of the inverted trough, thereby 
suggesting possible weaknesses in the diabatic parameterizations used in the NGM. B. Gross and J. Jusem discussed the interpretation of lee cyclogenesis as an isentropic potential vorticity (IPV) coupling mechanism using observed data and forecasts with a numerical model. A. van Delden concluded the session with a discussion of factors affecting squall-line formation and maintenance in western Europe.

\section{n. Session 10. Momentum transport by convection}

(Richard H. Johnson, chair)

In this session, momentum transport by convection was considered using both observational and theoretical approaches. Papers by W. Gallus and R. Johnson and by M. Jenkins and G. L. Zhang reported on computations of the momentum budget using rawinsonde data for the 10-11 June 1985 squall line of PRE-STORM. These studies found a pronounced mesolow in the midtroposphere in the trailing stratiform region that contributed to front-to-rear acceleration in the convective line and rear-to-front acceleration in the stratiform region. During the decaying stage, turbulent stresses in the convective line led to westerly acceleration in the lower to middle troposphere (with a maximum at midlevels), increasing the shear in the lower troposphere (an upgradient transport).

T. Matejka and M. LeMone presented a dual-Doppler radar analysis of momentum generation and redistribution in a Cooperative Convective Precipitation Experiment (CCOPE) squall line, which allowed determination of the contributions of individual terms to the total budget in unprecedented detail. The total momentum flux was partitioned into contributions from convective and stratiform updrafts and downdrafts, with convective updrafts being the primary contributors to the total flux. Individual cells in the convective line provided much of the flux. A strengthening of upward transport of front-to-rear momentum was noted as the system organized into a linear structure. Two CCOPE cases were compared and contrasted to previously analyzed cases by $\mathrm{M}$. LeMone, J. Fankhauser, and T. Matejka. One was characterized by moderate and one by strong westerly shear. In the strong westerly shear case, negative momentum flux was observed in the lower troposphere (contributed, in part, by the midlevel mesolow behind the convective line) and positive momentum flux occurred aloft. (Note that these momentum fluxes are in the line-normal direction, such that a positive flux is oriented in the direction of movement of the convective line.)

M. Moncrieff proposed a theory for two-dimensional squall-line circulations having flow configurations determined by nondimensional parameters that relate to Richardson and Froude numbers and to a measure of the work done by the pressure field within the storm. The analytical model offers some hope for parameterization of momentum transport in the linenormal direction of squall-line systems, where countergradient transports have been observed. The difficult task yet remains of how to relate the character of the cloud field (e.g., degree of convective organization, line orientation) to the grid values in a large-scale numerical model.

\section{o. Joint Session J2. Mesoscale phenomena and processes influenced by topography: II (Ronald B. Smith, chair)}

This session was concerned largely with the formation of eddies and other three-dimensional flow structures by mountains. The Denver eddy, a feature present during south and southeast flow, was discussed in two papers. E. Szoke and J. Augustine analyzed the climatology of the Denver eddy, while A. Crook considered the initiation of convection within it. The question of whether the Denver eddy is caused by lee effects of the Palmer Divide or blocking by the Front Range remains unresolved. C. Mass and M. Albright described a somewhat similar phenomenon: the Catalina eddy. The Catalina eddy forms in the Los Angeles Basin and over the adjacent sea during conditions of northerly flow. The feature may be viewed as a lee eddy in the shallow layer of cool marine air.

$K$. Ueyoshi described the numerical simulation of rain and lee eddy formation near Hawaii. A comparison of these results with the model simulations and aircraft observations of others shows broad agreement but differences in detail, suggesting opportunities for future work. In a unique study, H. Bluestein and S. Hrebenach described Doppler radar observations of an eddy standing above a region of complex terrain in the northern part of Taiwan. This eddy exists primarily between 4 and $7 \mathrm{~km}$ and may be stationary for a few hours.

D. Durran presented a numerical study of idealized nonrotating flow over a ridge with a gap. Surprisingly little tendency for accelerating flow through the gap was found, at least in comparison with the leeside downslope winds. S.-T. Soong described numerical results of airflow channeling in the Sacramento Valley during normal and heavy precipitation periods.

p. Session 11. Mesoscale convective systems: Structure, kinematics and dynamics (Margaret $A$. LeMone, chair)

The first two presentations dealt with mesoscale convective systems (MCSs) from a statistical point of view. B. Mapes and R. Houze used airborne Doppler radar data from the Equatorial Mesoscale Experiment (EMEX) to show how the mean convergence and 
hence vertical mass flux changes in a $22 \times 22 \mathrm{~km}$ area, as the convection changes from active to stratiform. Active convection was associated with deep convergence and positive vertical velocities through the 12 $\mathrm{km}$ sampled, while the transition and stratiform areas showed updraft above the middle troposphere and downdraft below. S. Rutledge noted that tropical convection from the Down Under Doppler and Electricity Experiment (DUNDEE) behaved similarly, and wind profiler data from the same experiment enabled documentation of subsidence ahead of convective bands. He characterized the convergence and divergence as weaker than that observed in PRE-STORM. W.-K. Tao, J. Simpson, M. McCumber, and S. Lang's emphasis was on the influence of external factors (radiation and ocean fluxes) and treatment of microphysics on the structure and evolution of numerically simulated storms. They found that the need for realistic treatment varied with the system; e.g., a simulated nonsquall Winter Monsoon Experiment (WMONEX) convective system required realistic treatment of radiation and surface fluxes for the development of heavy rainfall, while inclusion of these factors was less important for a squall line simulated from data from the Taiwan Area Mesoscale Experiment (TAMEX).

The many forms of mesoscale convective systems and their origins united the second set of papers. G. Tripoli, H. Cooper, and E. Smith presented a simulation of an Alabama (COHMEX) mesoscale convective system that evolved to form a supercell with an environmental sounding normally associated with smaller, less persistent storms. They hypothesized that the unusual behavior could be associated with the constructive interaction of the storm with storm-generated unbalanced flow. C. Leary, T. Bals, and J. Green showed how the complex reflectivity structure of a mesoscale system that occurred in PRE-STORM on 3-4 June 1985 related to the velocity and pressure fields. Among the interesting features - two strong vortices correlated with heavy precipitation, a strong wake low in the reflectivity-free notch, and a general association of higher reflectivity with upward-moving air. The low caused strong rearward acceleration at the surface. B. Smull and D. Jorgensen presented dual-Doppler syntheses of the wind, temperature, and pressure structure of this same low, showing that the lowest pressures lay beneath subsidence-warmed air. The retrieved pressure field at $0.5 \mathrm{~km}$ AGL agreed remarkably well with surface observations. R. McAnelly and W. Cotton used dual-Doppler data to document the structure and evolution of a PRE-STORM mesoscale convective system (third system on 3-4 June) with random rather than linear convection embedded. This system moved faster (25-30 $\left.\mathrm{m} \mathrm{s}^{-1}\right)$ than its constituent cells $\left(10-15 \mathrm{~m} \mathrm{~s}^{-1}\right)$ as a result of new cell formation in 200-km jumps (possibly related to a gravity-wave or wave-CISK mechanism). In spite of the chaotic reflectivity structure of the MCS, the system-relative circulation was well-organized, with ascending front-to-rear (east-to-west) flow at low to middle levels at and ahead (ENE) of the system. A descending rear-to-front branch developed in the SW quadrant of the system as convection weakened, combining with the ascending front-to-rear branch at higher levels and a front-to-rear flow at the lowest levels toward the WSW of the system to produce an ENE-WSW cross section resembling that across a convective band. M. Fortune and W. Cotton showed some examples of PRE-STORM mesoscale convective systems that resembled miniature frontal cyclones. One system shown (7 May) approached rotational balance; the authors showed an idealized "conveyor-belt model" of its flow. The formation of a mesoscale vortex in the stratiform regions of a 23-24 June PRE-STORM MCS was the subject of a talk by R. Johnson, S. Chen, G. Stumpf, and D. Bartels. The vortex extended from 4 to $8.5 \mathrm{~km}$, tilting slightly northeastward with height. Although the vortex was warm core, the warmest air was to the north of its center. Dry air at midlevels suggested that subsidence contributed significantly to vortex formation.

\section{q. Session 12. Mesoscale convective systems:}

Structure, kinematics and dynamics (continued from Session 11; Margaret A. LeMone, chair) This session focused on the MCS-generated mesovortex and circulations of larger scale. S. Chen and W. Frank described the formation of mesovortices in the stratiform regions of MCSs simulated by the Penn State/NCAR mesoscale model. These vortices, which under optimum conditions extend through the troposphere, are warm core at midlevels, and cold core at low $(p>900 \mathrm{mb})$ and high levels $(p<150-250$ $\mathrm{mb}$ ). Conditions favoring vortex development include an ambient trough and a strong rear-inflow jet within the MCS. Surface fronts are not favorable for development, and midlevel shear and dryness inhibit development. D. Raymond and $\mathrm{H}$. Jiang discussed these vortices qualitatively in terms of their associated potential vorticity (PV) distribution. The transport of mass from low to high levels by deep convection translates into a negative PV anomaly at high levels and positive PV anomaly at low levels, beneath which isentropes bulge upward. In the proper shear, the result is lifting of air at low levels by $500 \mathrm{~m}$ and more - sufficient to generate new convection. C. Tremback and W. Cotton described the modulation of a MCS by the mountain/ plains circulation. They found that east-west soil moisture differences were more important than topography in generating the circulation.

\section{r. Session 13. Boundary-layer circulations and cloud distributions (Peter R. Bannon, chair)}

This session focused on low-level phenomena strongly affected by surface processes. R. Pielke, G. Dalu, J. 
Weaver, J. Lee, and J. Purdom opened the session with a discussion of the impact of land use on mesoscale atmospheric circulations. They argued persuasively that land-use patterns, which alter the thermal and moisture properties of the lower atmosphere, need to be incorporated into regional modeling and forecasting studies. D. ReVelle, J. Logsdon, and A. Liu presented results of sensitivity studies of a boundarylayer model of the nocturnal low-level jet over flat terrain. The simulated jets were most sensitive to the Ekman number of the flow and the surface roughness. $H$. Shirer and T. Haack used a low-order nonlinear spectral model to assess the modification of the background wind and temperature profiles by roll vortices in the boundary layer. Good agreement with observations from the First International Satellite Cloud Climatology Project (ISCCP) Regional Experiment (FIRE) suggests that the observed rolls moved the temperature profile toward neutral stratification, while the shear of the cross-roll wind profile was increased. P. Sousounis and $\mathrm{H}$. Shirer presented a linear two-layer hydrostatic model with shear of the response to surface heating of a cold-air outbreak traversing a lake. The heating, incorporated as a mass sink, leads to subsidence when the incident flow is supercritical (Froude number greater than unity), suggestive of the cloud-free regions typically observed on the windward sides of the Great Lakes.

Two papers discussed the organization of convec- tion on the mesoscale. X.-Y. Huang and E. Källén, employing a nonlinear Boussinesq model with both liquid and vapor phases of water, demonstrated that the presence of external gravity waves can lead to the organization of convective elements into mesoscale clusters. B. Fielder addressed the problem of mesoscale cellular convection in the oceanic boundary layer using a nonlinear Boussinesq model. Cell broadening develops when the stress-free boundary conditions are thermally reinforcing so that the thermodynamic boundary fluxes are positively proportional to the thermal quantities. The results thus emphasized the importance of boundary entrainment.

H.-M. Hsu closed the session with a paper on the coupling of the coastal upwelling with the sea breeze. Using nonlinear, primitive equation models of both the ocean and atmosphere applied to a mesoscale canal geometry, strong coupling occurred only in the presence of a mean wind which amplified the diurnal fluctuation in the bulk aerodynamic surface stress.

Acknowledgments. Frances Huth of NCAR served as Local Arrangements Chair, providing exceptional administrative and technical support of the conference at every step of its planning and execution. During the conference, she was capably assisted by AMS volunteer Jim Vavrek, affiliated with the A. L. Spohn School, Hammond, Indiana. The program chair thanks Celeste lovinella and Sarah Marsh of the State University of New York at Albany for their numerous contributions in support of the administration of the conference. 